INPLASY

PROTOCOL

To cite: Liu et al. Chinese herbal medicine for hypertension complicated with hyperlipidemia: protocol for a systematic review and metaanalysis. Inplasy protocol 2020110144. doi:

10.37766/inplasy2020.11.0144

Received: 30 November 2020

Published: 30 November 2020

Corresponding author:

Yinan Liu

lyn1nı@qq.com

Author Affiliation:

Experimental research center,China academy of

Chinese medical sciences

Support: No.81673822 and ZZ2019012.

Review Stage at time of this submission: The review has not yet started.

Conflicts of interest: None.

\section{Chinese herbal medicine for hypertension complicated with hyperlipidemia: protocol for a systematic review and meta-analysis}

Liu, YN1; Liu, YQ2; Lei, Y3; Yang, J4; Wang, X5; Xiu, CK6; Hu, YH7; $\mathrm{Wu}, \mathrm{D}^{8} ; \mathrm{Wu}, \mathrm{Y}^{9}$.

Review question / Objective: P:Hypertension combined with hyperlipidemia(HTN-HLP); I:Chinese herbal medicine(CHM); C:Conventional pharmacotherapy, other CHM, other kind of TCM like acupuncture or no additional intervention to usual care; O:Blood pressure, blood lipids, TCM symptom score, total clinical efficacy rate and adverse events; S: Randomized Controlled Trials (RCTs).

Condition being studied: Hypertension combined with hyperlipidemia (HTN-HLP), as a common clinical chronic disease combination, will increase the incidence of cardiovascular and cerebrovascular diseases, increase the occurrence of sudden death and other adverse events. At present, the commonly used therapeutic drugs are mainly combined with antihypertensive drugs and lipid-lowering drugs, which not only have poor compliance, but also have adverse reactions. Currently, traditional Chinese medicine (TCM), as a traditional medicine in China, has been applied in clinical practice for thousands of years and has rich clinical experience in treating HTN-HLP. However, there is no systematic evaluation of the efficacy, safety and improvement of patients' quality of life. This systematic review and metaanalysis will assess studies of the effects and safety of Chinese herbal medicine(CHM) for HTN-HLP patients.

INPLASY registration number: This protocol was registered with the International Platform of Registered Systematic Review and Meta-Analysis Protocols (INPLASY) on 30 November 2020 and was last updated on 30 November 2020 (registration number INPLASY2020110144).

\section{INTRODUCTION}

Review question / Objective: $P$ : Hypertension combined with hyperlipidemia (HTN-HLP); I:Chinese herbal m edic ine ( C H M ); C: C onventional pharmacotherapy, other CHM, other kind of TCM like acupuncture or no additional 
intervention to usual care; O:Blood pressure, blood lipids, TCM symptom score, total clinical efficacy rate and adverse events; S: Randomized Controlled Trials (RCTs).

Condition being studied: Hypertension combined with hyperlipidemia (HTN-HLP), as a common clinical chronic disease combination, will increase the incidence of cardiovascular and cerebrovascular diseases, increase the occurrence of sudden death and other adverse events. At present, the commonly used therapeutic drugs are mainly combined with antihypertensive drugs and lipid-lowering drugs, which not only have poor compliance, but also have adverse reactions. Currently, traditional Chinese medicine (TCM), as a traditional medicine in China, has been applied in clinical practice for thousands of years and has rich clinical experience in treating HTNHLP. However, there is no systematic evaluation of the efficacy, safety and improvement of patients' quality of life. This systematic review and meta-analysis will assess studies of the effects and safety of Chinese herbal medicine (CHM) for HTNHLP patients.

\section{METHODS}

Participant or population: The adult patients (18 years of age and older) were diagnosed as HTN-HLP. HTN should be confirmed according to the " 2018 Chinese guidelines for the management of hypertension" and HLP should be confirmed according to the standard diagnostic criteria including the "Guidelines for the prevention and treatment of dyslipidemia in Chinese adults (2016)". There will be no limited to gender, age, ethnicity and country. Children, pregnant women, patients with secondary hypertension and combined with other complications will be exclude.

Intervention: We will consider the intervention used clinically common CHM, like Huanglian Wendan Decoction, Banxia Baizhu Tianma Decoction, Xuefuzhuyu Decoction, Huanglian jiedu decoction, and
Tianma Gouteng decoction, which were used alone or in combination. There is no limit to duration, dose or route of administration.

Comparator: Treatments in the control group can be conventional pharmacotherapy, other CHM, other kind of TCM like acupuncture or no additional intervention to usual care. If combined treatment of CHM and other therapy were used in the experimental group. The same therapy must be used in the control group.

Study designs to be included: Literature search, literature screening, data collecton, software analysis, discussion and conclusion.

Eligibility criteria: Five main factors of PICOS were used for this research: (1) Participant (P); (2) Intervention (I); (3) Comparator (C); (4) Outcome (O); (5) Study design (S).

Information sources: We will systematically search the published reports on RCTs throughout eight databases: Pubmed, Embase, Cochrane Central Register of Controlled Trials, Web of Science, China National Knowledge Infrastructure, Wan fang Database, VIP Information and SinoMed from the establishment of each database to 01, February 2021. No restriction on publication language and status was preset. Otherwise, we will also manually retrieve literature from the WHO Trial Register, Google Scholar, Chinese Clinical Trial Register, Baidu and other search engines to acquire other unpublished articles.

Main outcome(s): Blood pressure: including systolic blood pressure(SBP), diastolic blood pressure (DBP); Blood lipids: including total cholesterol(TC), triglyceride(TG), low-density lipoprotein (LDL-C), high-density lipoprotein (HDL-C), apolipoprotein A (apo A), apolipoprotein A(apo B) and so on; TCM Symptom Score; Total clinical efficacy rate: According to the Guiding Principles for Clinical Research of Drugs: (1) markedly effective: DBP reached the normal range and decreased $>10 \mathrm{mmHg}$ 
or DBP did not fall to normal, but it had decreased 20mmHg; TC decreased $\geq 20 \%$ or TG decreased $\geq 40 \%$ or HDL-C increased $\geq 0.26 \mathrm{mmol} / \mathrm{L}$; (2) effective:DBP reached the normal range and decreased $30 \mathrm{mmHg}$;TC decreased by $10 \%$ to $20 \%$ or TG decreased by $20 \%$ to $40 \%$ or HDL-C increased by 0.10 to $0.26 \mathrm{mmol} / \mathrm{L}$; (3) invalid: did not meet the above criteria. In this study, Clinical total effective rate(CTER)=[(total number of patients-invalid)/ total number of patients] ${ }^{\star} 100 \%$.

Quality assessment / Risk of bias analysis: Two researchers will independently assessed the risk of bias in the eligible studies through the risk assessment tool for bias in the Cochrane Handbook version 5.1.0. The evaluation is mainly made from seven aspects:(1) random sequence generation(selection bias);(2) allocation concealment (selection bias);(3) blinding of participants and personnel(performance bias);(4) blinding of outcome data(attrition bias);(5) incomplete outcome data (attrition bias);(6) selective reporting(reporting bias); (7) Other biases.And it evaluates the seven aspects according to "low risk", "not clear" and "high risk".

Strategy of data synthesis: Meta-analysis will be processed by Review Manager software(Version 5.3, Copenhagen: The Nordic Cochrane Center, 2014). Dichotomous outcome was expressed by risk ratio(RR), continuous data as mean difference (MD), both with $95 \%$ confidence interval(CI). $\mathrm{P0.1}$ and $1250 \%$, there is heterogeneity, subgroup analysis will be performed to investigate the sources of heterogeneity. If the subgroup analysis results show that there is sufficient similarity between the subgroups $(P>0.1$, $12<50 \%$ ), the fixed-effect model will be used for meta-analysis; otherwise, if there is a statistical heterogeneity but no clinical heterogeneous temperament between subgroups, the random-effects model will be conducted. The subgroup or sensitivity analyses, or only descriptive analyses will be performed if significant clinical heterogeneity exists.
Subgroup analysis: When conducting metaanalysis, for each outcome studies will be grouped according to: (1) The type of treatment (Huanglian Wendan Decoction, Banxia Baizhu Tianma Decoction, Xuefuzhuyu Decoction, Huanglian jiedu decoction, and Tianma Gouteng decoction, et al); (2) The comparator (no treatment, general treatment or other kind of CTM).

Sensibility analysis: Sensitivity analysis will be performed according to clinical factors(age, gender, Chinese medicine syndrome), intervention method (Huanglian Wendan Decoction, Banxia Baizhu Tianma Decoction, Xuefuzhuyu Decoction, Huanglian jiedu decoction, and Tianma Gouteng decoction) or methodological heterogeneity(sample size, risk of bias).

Language: No limit to language.

Country(ies) involved: China.

Keywords: Chinese herbal medicine, hypertension complicated with hyperlipidemia, protocol, systematic review.

Contributions of each author:

Author 1 - Yinan Liu.

Author 2 - Yiqing Liu.

Author 3 - Yan Lei.

Author 4 - Jing Yang.

Author 5 - Xue Wang.

Author 6 - Chengkui Xiu.

Author 7 - Yanhong Hu.

Author 8 - Dan Wu.

Author 9 - Ye Wu. 